\title{
Correcting Fe- and S-XANES beam damage and recognizing rapid redox equilibration of olivine-hosted melt inclusions
}

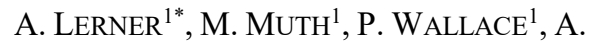 \\ LANZIROTTI $^{2}$, M. NEWVILLE ${ }^{2}$, G. GAETANI ${ }^{3}$, P. \\ $\mathrm{CHOWDHURY}^{4}$, R. DASGUPTA ${ }^{4}$ \\ ${ }^{1}$ University of Oregon, Eugene, OR \\ (*correspondence: alerner@uoregon.edu) \\ 2 The University of Chicago, Chicago, IL \\ ${ }^{3}$ Woods Hole Oceanographic Institution, Woods Hole, MA \\ ${ }^{4}$ Rice University, Houston, TX
}

The redox state of melts influences crystallization, partitioning, and degassing behavior of magma. Synchrotronbased XANES is a powerful tool to calculate redox states by measuring multivalent elements. The small beam size of XANES makes it a valuable technique for determining redox states of melt inclusions (MI). However, the small size of MI and the high penetration and beam damage potential of $\mathrm{X}$ rays makes XANES measurements in MI challenging.

We present observations of rapid Fe- and S-XANES beam damage in olivine-hosted MI from Kilauea volcano and the southern Cascades. We develop approaches to recognize and mitigate beam damage through repeated rapid analyses. Using rapid scans and applying a time-dependent correction to Fe-XANES pre-edge centroid positions, we correct to undamaged initial $\mathrm{Fe}^{3+} / \Sigma \mathrm{Fe}$ speciation in MI. We similarly apply repeat rapid scans to recognize S-XANES beam damage and we develop a peak fitting method and correction factor to restore initial $\mathrm{S}^{6+} / \Sigma \mathrm{S}$ speciation. Using these approaches, we can confidently measure $\mathrm{Fe}$ and $\mathrm{S}$ speciation even in beam damage-susceptible MI.

However, accurately interpreting redox measurements of MI require further considerations of a variety of processes that can affect inclusions. We observe rapid atmospheric oxidation (up to $+2 \log$ units over hours to days) of olivinehosted MI in a sub-aerial lava channel from the 2018 eruption of Kilauea. The observations of rapid redox re-equilibration of olivine-hosted MI are consistent with modeled timescales of metal vacancy diffusion through olivine. We therefore conclude that olivine-hosted MI are susceptible to rapid redox re-equilibration. Depending on the timescales of magma ascent and quenching, measured redox conditions in MI may reflect conduit or emplacement conditions rather than melt conditions at the time of inclusion entrapment. While we have developed ways to accurately measure redox conditions in MI by XANES, petrologic interpretation of such redox measurements requires additional careful consideration. 